\title{
New spatial mechanisms for the kinematic analysis of the tibiotalar joint R Franci $^{2}$, V Parenti-Castelli*1, C Belvedere ${ }^{2}$ and A Leardini ${ }^{2}$
}

\author{
Address: ${ }^{1}$ Department of Mechanical Engineering, University of Bologna, Italy and ${ }^{2}$ Movement Analysis Laboratory, Istituto Ortopedici Rizzoli, \\ Italy \\ Email: V Parenti-Castelli* - vincenzo.parenticastelli@mail.ing.unibo.it \\ * Corresponding author
}

from Ist Congress of the International Foot \& Ankle Biomechanics (i-FAB) community

Bologna, Italy. 4-6 September 2008

Published: 26 September 2008

Journal of Foot and Ankle Research 2008, I(Suppl I):O44 doi:I0.I I86/I757-I I46-I-SI-O44

This abstract is available from: http://www.jfootankleres.com/content/I/SI/O44

(c) 2008 Franci et al; licensee BioMed Central Ltd.

\section{Introduction}

In virtually unloaded conditions, the tibiotalar (ankle) joint behaves as a single degree-of-freedom system, and two fibres within the calcaneal-fibular and tibio-calcaneal ligaments remain nearly isometric throughout the flexion arc [1]. A relevant theoretical model also showed that three articular surfaces and two ligaments act together as a mechanism to control the passive kinematics [2]. Two equivalent spatial parallel mechanisms were formulated, with ligament fibres assumed isometric and articulating surfaces assumed rigid, either as three sphere-plane contacts, or as a single spherical pair. Predicted and measured motion in three specimens compared fairly well. Important enhancement of this previous work is here presented, with more accurate experimental data, more anatomical model surfaces, and a more robust mathematical model.

\section{Methods}

Four fresh frozen amputated legs, free of anatomical defects, were analysed under passive conditions [1]. Motion of tibia, fibula, talus and calcaneus and geometrical arrangement of the main articular surfaces and ligament attachments were obtained by a camera-based Knee Navigation System (Stryker ${ }^{\circledast}$, Kalamazoo, MI-USA).

A new equivalent spatial mechanism was based on three sphere-to-sphere contacts (two between the trochlea tali and tibial mortise, one between the lateral talus and the lateral malleolus) and two rigid fibres. This one degree of freedom mechanism was represented also by 5 rigid links connecting 5 points on the tibia-fibula to 5 points on the talus-calcaneus segments ('5-5' model) [3]. Because measured motion was nearly spherical, contact was also modelled as a spherical wrist ('s-w' model) [4].

For each model, a bounded optimization procedure was used to find the optimal geometric parameter set which makes it possible to obtain the best-fit between experimental and model motion. For each of the four specimens, initial tentative values for the geometrical parameters were obtained from the experiments in-vitro.

\section{Results}

Experimental motion compared very well with corresponding simulations from ' $5-5$ ' model, just a little worse from ' $s-w$ ' (Figure 1). The difference between initial tentative and corresponding final optimal ligament fibre attachments was found small $(2-8 \mathrm{~mm})$. The difference for the sphere centres was larger, accounted for the limited digitised area.

\section{Conclusion}

The proposed models replicated well ankle passive motion, still reflecting at the same time the main anatomical structures of this joint. These models are believed to be very useful tools for ankle surgical procedures simulation and planning, as well as for ankle prosthesis design. 

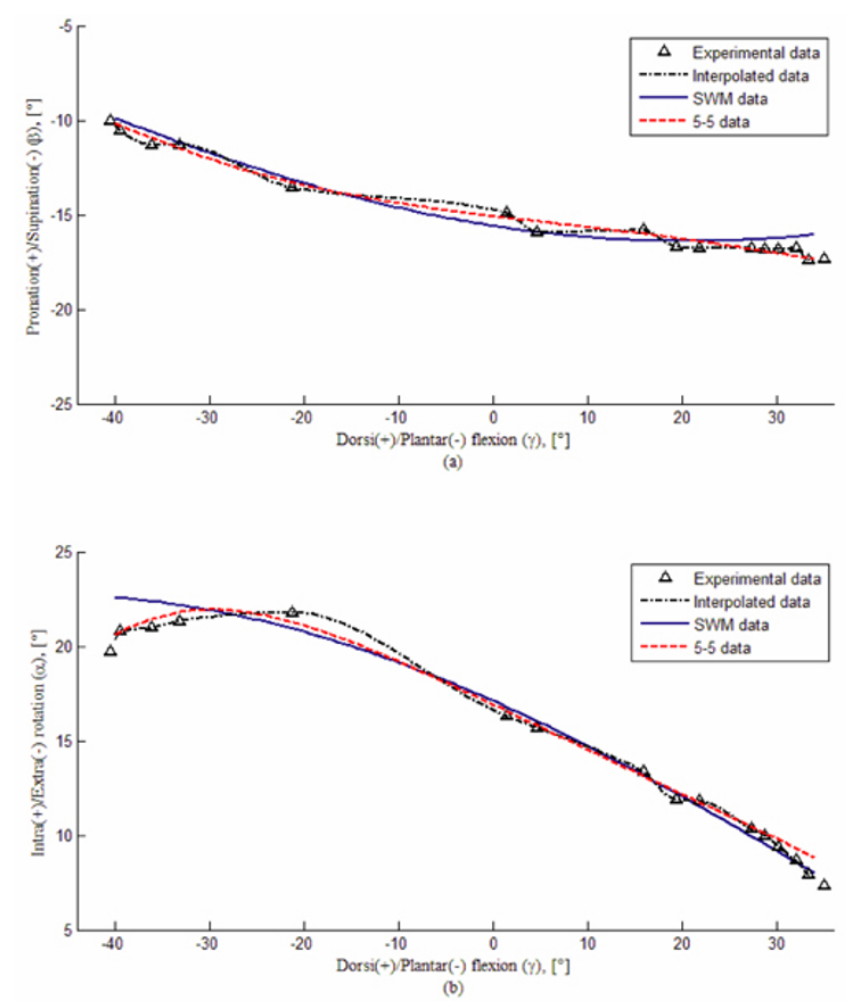

\section{Figure I}

Experimental (black) joint rotations out-of-sagittal plane vs dorsi/platar flexion superimposed to corresponding model '5-5' (red) and 's-w' (blue) predictions.

\section{References}

I. Leardini A, et al: J Biom 1999, 32: I I I-II8.

2. Di Gregorio R, et al.: Med Biol Eng Comput 2007, 45(3):305-13.

3. Franci R, et al.: Proceedings of the ASME IDETC/CIE, Las Vegas, Nevada, USA 2007.

4. Franci R, et al.: IAK, Lima, Peru; 2008. 